\title{
Dipyridine cobalt chloride: a novel and efficient catalyst for the synthesis of 14-aryl $14 H$-dibenzo[a.j] xanthenes under solvent-free conditions
}

\author{
J. Venu Madhav, B. Suresh Kuarm, and B. Rajitha* \\ Department of Chemistry, National Institute of Technology, Warangal 506 004, India. \\ E-mail: rajitabhargavi@yahoo.com
}

\begin{abstract}
A convenient procedure for the synthesis of 14-aryl 14H-dibenzo[a.j]xanthenes is reported using the condensation of $\beta$-naphthol with aryl and chromone aldehydes in the presence of dipyridine cobalt chloride as the catalyst under solvent-free conditions.
\end{abstract}

Keywords: Xanthene, aldehydes, $\beta$-naphthol, $\mathrm{CoPy}_{2} \mathrm{Cl}_{2}$, solvent-free conditions

\section{Introduction}

The preparation of benzoxanthenes is important due to their broad spectrum of biological and therapeutic properties such as antiviral, ${ }^{1}$ antibacterial, ${ }^{2}$ and antiinflammatory ${ }^{3}$ activities and efficiency in photodynamic therapy, ${ }^{4}$ and in antagonism of the paralyzing action of zoxazolamine. ${ }^{5}$ Further, these compounds can be employed as dyes, ${ }^{6} \mathrm{pH}-$ sensitive fluorescent materials for visualization of biomolecules ${ }^{7}$ and in laser technologies. ${ }^{8}$ For the construction of xanthenes and benzoxanthenes, various procedures are available including the cycloacylation of carbamates, ${ }^{9}$ trapping of benzynes by phenol, ${ }^{10}$ cyclocondensation between 2 -hydroxy aromatic aldehydes and 2-tetralone, ${ }^{11}$ cyclodehydrations ${ }^{12}$ and intramolecular phenyl carbonyl reaction of aldehydes with $\beta$-naphthol by dehydration. ${ }^{13}$ Other routes for the synthesis of benzoxanthenes and related products include the reaction of $\beta$-naphthol with formamide, ${ }^{14}$ carbon monoxide ${ }^{15}$ and 2-hydoxynaphth-1-ylmethanol. ${ }^{16}$ Various reagents have been employed for the synthesis of benzoxanthenes such as $\mathrm{Al}\left(\mathrm{HSO}_{4}\right)_{3},{ }^{17}$ sulfamic acid, ${ }^{18}$ Selectfluor ${ }^{\mathrm{TM}},{ }^{19}$ molecular iodine, ${ }^{20}$ Amberlyst- $15,{ }^{21}$ hydrochloric acid or phosphoric acid, ${ }^{22} \mathrm{H}_{2} \mathrm{SO}_{4}$ in acetic acid as solvent, ${ }^{23}$ and $p$ $\mathrm{TSA}^{24}$ silica sulfuric acid ${ }^{25}$ and cation-exchange resins ${ }^{26}$ as catalysts. However, many of these methods suffer from some limitations, such as low yields, prolonged reaction time, tedious workup procedures and co-occurrence of side reactions. Some of the catalysts are destroyed in the work-up procedure and cannot be recovered or reused. Therefore, the search continues for a 
better catalyst for the synthesis of benzoxanthenes in terms of operational simplicity, reusability of catalyst, low cost and greater selectivity.

\section{Results and Discussion}

In continuation of our search for new catalysts, ${ }^{27}$ we report in this paper the synthesis of benzoxanthenes via the condensation of aldehydes with $\beta$-naphthol promoted by dipyridine cobalt chloride under solvent-free conditions (Scheme 1). The catalyst is solid (violet color crystals), soluble in water, stable in air, and immiscible in common organic solvents; it is reusable and has high thermal stability. In addition, its toxicity is low.

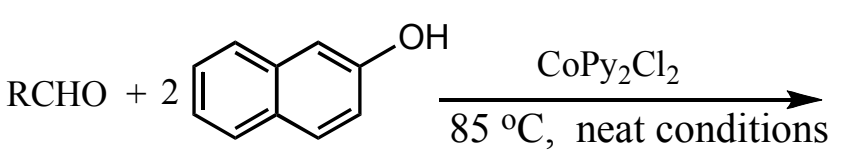

1

2<smiles>[R]C1c2c(ccc3ccccc23)Oc2ccc3ccccc3c21</smiles>

3

$\mathrm{R}=$ Aryl, chromone

\section{Scheme 1}

The reaction was carried out at $85{ }^{\circ} \mathrm{C}$ temperature for various times (Table 1) using a 2:1 mol ratio mixture of $\beta$-naphthol and the aldehyde in the presence of $\mathrm{CoPy}_{2} \mathrm{Cl}_{2}(0.1 \mathrm{mmol})$ to give the products in excellent yields. 
Table 1. Dipyridine cobalt chloride catalysed synthesis of 14-aryl 14Hdibenzo[a,j]xantenes 3

\begin{tabular}{|c|c|c|c|c|}
\hline Entry & Aldehyde & Time(h) & 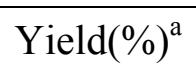 & $\mathrm{Mp}\left({ }^{\circ} \mathrm{C}\right)$ \\
\hline 1 & & 2 & 97 & $183^{12 b}$ \\
\hline 2 & & 2.5 & 93 & $228^{12 \mathrm{~b}}$ \\
\hline 3 & & 2 & 95 & $287^{12 c}$ \\
\hline 4 & & 3 & 92 & $215^{12 \mathrm{c}}$ \\
\hline 5 & & 2 & 96 & $296^{12 b}$ \\
\hline 6 & & 2.5 & 93 & $190^{12 c}$ \\
\hline 7 & & 2.5 & 96 & $312^{12 b}$ \\
\hline 8 & & 3 & 92 & $293^{18}$ \\
\hline 9 & & 1.5 & 92 & $140^{19}$ \\
\hline 10 & & 2 & 94 & $205^{12 \mathrm{c}}$ \\
\hline 11 & & 3 & 93 & $258^{12 \mathrm{c}}$ \\
\hline 12 & & 2 & 94 & $238^{12 c}$ \\
\hline 13 & & 2.5 & 95 & $258-259^{18}$ \\
\hline 14 & & 3 & 94 & $213^{12 c}$ \\
\hline 15 & & 6 & 72 & 195 \\
\hline 16 & & 8 & 65 & 125 \\
\hline
\end{tabular}

${ }^{a}$ Yields refer to isolate pure products and which were characterized by NMR, IR and mass spectral data and by comparison with those of authentic samples. 


\section{Experimental Section}

General Procedures. All the melting points were uncorrected. The progress of the reaction was monitored by (TLC). IR spectra (KBr) were recorded on Shimadzu FTIR model 8010 spectrometer and the ${ }^{1} \mathrm{H}$ NMR spectra on Varian Gemini $200 \mathrm{MHz}$ spectrometer using TMS as the internal standard. The $\mathrm{C}, \mathrm{H}$, and $\mathrm{N}$ analysis of the compound was done on a Carlo Erba model EA1108. Mass spectra were recorded on a JEOL JMS D-300 Spectrometer.

\section{Typical procedure}

A mixture of the aldehyde $(1 \mathrm{mmol})$ and $\beta$-naphthol $(2 \mathrm{mmol})$ was well stirred with $\mathrm{CoPy}_{2} \mathrm{Cl}_{2}$ $(0.1 \mathrm{mmol})$ at $85{ }^{\circ} \mathrm{C}$ for the appropriate time according to Table. After completion of the reaction, the reaction contents were cooled to room temperature, water $(3 \mathrm{ml})$ was added and the mixture stirred for $2 \mathrm{~min}$. The solid obtained was separated by filtration and recrystallized from ethanol. After filtration, the filtrate (water) containing the catalyst could be evaporated under reduced pressure to give a violet solid. The recovered catalyst was washed with ether, dried at 85 ${ }^{\circ} \mathrm{C}$ for $2 \mathrm{~h}$ under high-pressure prior to use in the other reaction.

\section{Selected product data}

14-(4-Chlorophenyl)-14H-dibenzo[a,j] xanthene (Table 1, entry 3). Brown solid: mp $287^{\circ} \mathrm{C}$. IR $\left(\mathrm{KBr}, \mathrm{cm}^{-1}\right): 3133,1618,1590,1450,1225,1105,820,776 ;{ }^{1} \mathrm{H}$ NMR $\left(\mathrm{CDCl}_{3}\right): \delta 6.50(\mathrm{~s}$, $1 \mathrm{H}), 7.07-8.31(\mathrm{~m}, 16 \mathrm{H}) ;{ }^{13} \mathrm{C} \mathrm{NMR}\left(75 \mathrm{MHz}, \mathrm{CDCl}_{3}\right): \delta 36.7,117.8,118.5,124.2,125.5,127.9$, 129.2, 129.5, 130.1, 130.5, 131.5, 131.6, 131.8, 145.3, 148.8; EIMS, $70 \mathrm{Ev}, m / z: 392\left(\mathrm{M}^{+}\right), 281$, 268; Anal. Calcd for $\mathrm{C}_{27} \mathrm{H}_{17} \mathrm{ClO}$ : C, 82.54; H, 4.36\%; Found: C, 82.52; H, 4.33\%.

14-(2-Nitrophenyl)-14H-dibenzo[a,j]xanthene (Table 1, entry 8). Yellow solid: mp $293{ }^{\circ} \mathrm{C}$. IR $\left(\mathrm{KBr}, \mathrm{cm}^{-1}\right): 3400,3056,1593,1522,1351,1240,1142,807,746 ;{ }^{1} \mathrm{H}$ NMR $\left(\mathrm{CDCl}_{3}\right): \delta 7.52(\mathrm{~s}$, 1H), 7.10-8.54 (m, 16H); ${ }^{13} \mathrm{C}$ NMR (75 MHz, $\left.\mathrm{CDCl}_{3}\right): \delta=32.7,118.0,118.3,123.2,124.5,125.1$, 125.3, 127.7, 128.0, 129.3, 129.6, 129.9, 130.6, 132.2, 132.6, 134.4, 141.2, 147.5, 149.8; EIMS, 70Ev, $m / z$ : $403\left(\mathrm{M}^{+}\right), 281,268$; Anal. Calcd for $\mathrm{C}_{27} \mathrm{H}_{17} \mathrm{NO}_{3}$ : C, 80.38; H, 4.25; N, 3.47\%; Found: C, $80.25 ; \mathrm{H}, 4.23 ; \mathrm{N}, 3.57 \%$.

14-(4-Hydroxyphenyl)-14H-dibenzo[a,j]xanthene (Table 1, entry 9). Pink solid: mp $140{ }^{\circ} \mathrm{C}$. IR $\left(\mathrm{KBr}, \mathrm{cm}^{-1}\right): 3400,1581,1511,1405,1250,1241,814 ;{ }^{1} \mathrm{H} \mathrm{NMR}\left(\mathrm{CDCl}_{3}\right): \delta 4.95$ (br s, $1 \mathrm{H}$, $\mathrm{OH}), 6.42(\mathrm{~s}, 1 \mathrm{H} \mathrm{CH}), 6.58-8.39(\mathrm{~m}, 16 \mathrm{H}) ;{ }^{13} \mathrm{C} \mathrm{NMR}\left(75 \mathrm{MHz}, \mathrm{CDCl}_{3}\right): \delta 37.5,115.8,117.6$, 118.4, 123.3, 124.6, 127.6, 129.2, 129.3, 129.9, 131.4, 131.9, 137.9, 149.2, 154.2; EIMS, 70 Ev, m/z: $374\left(\mathrm{M}^{+}\right), 281,268$; Anal. Calcd for $\mathrm{C}_{27} \mathrm{H}_{18} \mathrm{O}_{2}$ : C, 86.61; H, 4.85\% Found: C, 86.62; H, $4.81 \%$.

14-(3-Fluorophenyl)-14H-dibenzo[ $[a, j]$ xanthene (Table 1, entry 13). Brown solid: mp 258-259 ${ }^{\circ} \mathrm{C}$. IR $\left(\mathrm{KBr}, \mathrm{cm}^{-1}\right): 3153,1594,1403,1240,1206,1067,817,745 ;{ }^{1} \mathrm{H}$ NMR $\left(\mathrm{CDCl}_{3}\right): \delta 6.51(\mathrm{~s}$, $1 \mathrm{H}), 6.72-8.35(\mathrm{~m}, 16 \mathrm{H}) ;{ }^{13} \mathrm{C} \mathrm{NMR}\left(75 \mathrm{MHz}, \mathrm{CDCl}_{3}\right): \delta 38.2,113.8$ and $90,114.0\left(J_{\mathrm{C}-\mathrm{F}} 21.5\right.$ $\mathrm{Hz}), 115.4$ and $115.8\left(J_{\mathrm{C}-\mathrm{F}} 21.5 \mathrm{~Hz}\right), 117.2,118.2,122.7,124.31$, and $124.32\left(J_{\mathrm{C}-\mathrm{F}} 2.8 \mathrm{~Hz}\right) 124.7$, $127.3,129.3,129.5,130.1$ and130.2 $\left(J_{\text {C-F }} 8.3 \mathrm{~Hz}\right), 131.4,131.7\left(J_{\mathrm{C}-\mathrm{F}} 19.4 \mathrm{~Hz}\right), 147.8,147.9\left(J_{\mathrm{C}-\mathrm{F}}\right.$ 
6.2 Hz), 149.2, 161.7, 165.1; EIMS, $70 \mathrm{Ev}, \mathrm{m} / z: 376\left(\mathrm{M}^{+}\right)$, 281, 268; Anal. Calcd for $\mathrm{C}_{27} \mathrm{H}_{17} \mathrm{FO}$ : C, 86.15; H, 4.55; F, 5.05\%; Found: C, 86.12; H, 4.53; F, 5.06\%.

3-(14H-Dibenzo[a,j]xanthen-14-yl)chromen-4-one: (Table 1, entry 15). Light yellow solid: $\mathrm{mp} 195{ }^{\circ} \mathrm{C}$. IR $\left(\mathrm{KBr}, \mathrm{cm}^{-1}\right): 3075,1617,1594,1407,1235,1208,1060,819,740 ;{ }^{1} \mathrm{H}$ NMR $\left(\mathrm{CDCl}_{3}\right): \delta 6.10(\mathrm{~s}, 1 \mathrm{H}$ chromone 2-H), $6.42(\mathrm{~s}, 1 \mathrm{H}), 7.24-7.96(\mathrm{~m}, 16 \mathrm{H}) ; \mathrm{EIMS}, 70 \mathrm{Ev}, \mathrm{m} / z: 426$ $\left(\mathrm{M}^{+}\right)$; Anal. Calcd for $\mathrm{C}_{30} \mathrm{H}_{18} \mathrm{O}_{3}$ : C, 84.49; H, 4.25\%; Found: C, 84.42; H, 4.29\%.

3-(14H-Dibenzo[a,j]xanthen-14-yl)-6-nitrochromen-4-one: (Table 1, entry 16). Brown solid: mp $125{ }^{\circ} \mathrm{C}$. IR $\left(\mathrm{KBr}, \mathrm{cm}^{-1}\right): 3151,1635,1590,1403,1240,1206,1067,817,745 ;{ }^{1} \mathrm{H}$ NMR $\left(\mathrm{CDCl}_{3}\right): \delta 6.15(\mathrm{~s}, 1 \mathrm{H}$ chromone 2-H), $6.51(\mathrm{~s}, 1 \mathrm{H}), 7.31-8.10(\mathrm{~m}, 15 \mathrm{H})$; EIMS, $70 \mathrm{Ev}, \mathrm{m} / z: 471$ $\left(\mathrm{M}^{+}\right)$; Anal. Calcd for $\mathrm{C}_{30} \mathrm{H}_{17} \mathrm{NO}_{5}$ : C, 76.43; H, 3.63; N, 2.97\%; Found: C, 76.47; H, 3.61; N, $2.94 \%$.

\section{References}

1. Lambert, R. W.; Martin, J. A.; Merrett, J. H.; Parkes, K. E. B.; Thomas, G. J., PCT Int. Appl. WO 9706178, 1997; Chem. Abstr. 1997, 126, p212377y.

2. Hideo, T. Chem. Abstr. 1981, 95, 80922b, Jpn. Tokkyo Koho JP 56005480,1981.

3. Poupelin, J. P.; Saint-Ruf, G.; Foussard-Blanpin, O.; Narcisse, G.; Uchida-Ernouf, G.; Lacroix, R. Eur. J. Med. Chem. 1978, 13, 67.

4. (a) Ion, R. M. Prog. Catal. 1997, 2, 55. (b) Ion, R. M.; Frackowiak, D.; Planner, A.; Wiktorowicz, K. Acta Biochim. Pol. 1998, 45, 833.

5. (a) Saint-Ruf, G.; De, A.; Hieu, H. T. Bull. Chim. Ther. 1972, 7, 83. (b) Saint-Ruf, G.; Hieu, H. T.; Poupelin, J. P. Naturwissenschaften 1975, 62, 584.

6. (a) Menchen, S. M.; Benson, S. C.; Lam, J. Y. L.; Zhen, W.; Sun, D.; Rosenblum, B. B.; Khan, S. H.; Taing, M., U.S. Patent, US6583168, 2003; Chem. Abstr. 2003, 139, p54287f. (b) Banerjee, A.; Mukherjee, A. K. Stain Technol. 1981, 56, 83-85. (c) Reynolds, G. A.; Tuccio, S. A.; Peterson, O. G.; Specht, D. P. Chem. Abstr. 1971, 71, p81334c, Ger. Offen. DE2109040, 1971.

7. Knight, C. G.; Stephens, T. Biochem. J. 1989, 258, 683.

8. (a) Sirkeeioglu, O.; Talinli, N.; Akar, A. J. Chem. Res. (S) 1995, 502. (b) Ahmad, M.; King, T. A.; Ko, Do-K.; Cha, B. H.; Lee, J. J. Phys. D: Appl. Phys. 2002, 35, 1473.

9. Quintas, D.; Garcia, A.; Dominguez, D. ChemInform 2004, 35(14), 154.

10. (a) Knight, D. W.; Little, P. B. Synlett 1998, 1141; (b) Knight, D. W.; Little, P. B. J. Chem. Soc., Perkin Trans. I 2001, 1771.

11. Jha, A.; Beal, J. Tetrahedron Lett. 2004, 45, 8999-9001.

12. (a) Bekaert, A.; Andrieux, J.; Plat, M. Tetrahedron Lett. 1992, 33, 2805. (b) Sarma, R. J.; Baruah, J. B. Dyes Pigm. 2005, 64, 91. (c) Buehler, C. A.; Cooper, D. E.; Scrudder, E. O. J. Org. Chem. 1943, 8, 316. 
13. (a) Van Allan, J. A.; Giannini, D. D.; Whitesides, T. H. J. Org. Chem. 1982, 47, 820. (b) Ohishi, T.; Kojima. T.; Matsuoka, T.; Shiro, M.; Kotsuki, H. Tetrahedron Lett. 2001, 42, 2493. (c) Khoramabadizad, A.; Kazemi, Z.; Amin Rudbari, H. J. Korean Chem. Soc. 2002, $46,541$.

14. Papini, P.; Cimmarusti, R. Gazz. Chim. Ital. 1947, 77, 142.

15. Ota, K.; Kito, T. Bull. Chem. Soc. Jpn. 1976, 49, 1167.

16. Sen, R. N.; Sarkar, N. J. Am. Chem. Soc. 1925, 47, 1079.

17. Shaterian, H. R.; Ghashang, M.; Mir, N. ARKIVOC 2007, (xv), 1.

18. Rajitha, B.; Sunil Kumar, B.; Thirupathi Reddy, Y.; Narsimha Reddy, P.; Sreenivasulu, N. Tetrahedron Lett. 2005, 46, 8691.

19. Kumar, P. S.; Sunil Kumar, B.; Rajitha, B.; Narsimha Reddy, P.; Sreenivasulu, N.; Thirupathi Reddy, Y. ARKIVOC 2006, (xii), 1.

20. Biswanath Das.; Ravikanth, B.; Ramu, R.; Laxminarayana, K.; Vittal Rao, B. J. Mol. Catal. A: Chem. 2006, 255, 74.

21. Ko, S.; C. F Yao. Tetrahedron Lett. 2006, 47, 8827.

22. Khorramabadi-zad, A.; Kazemi, Z.; Amini Rudbari, H. J. Korean Chem. Soc. 2002, 46, 541.

23. Sarma, R. J.; Baruah, J. B. Dyes pigm. 2005, 64, 91.

24. (a) Khorramabadi-zad, A.; Akbari, S. A.; Shiri, A.; Veisi, H. J. Chem. Res. 2005, 277. (b) Khosropour, A. R.; Khodaei, M. M.; Moghhannian, H. Synlett. 2005, 955.

25. H. R. Shaterian, M. Ghashang, Hassankhani, A. Dyes Pigm. 2008, 76, 564.

26. Patil, S. B.; Bhat, R. P.; Samant, S. D. Synthetic Commun. 2006, 36, 2163.

27. (a) Rajitha, B.; Naveen Kumar, V.; Someshwar, P.; Venu Madhav, J.; Narsimha Reddy, P.; Thirupathi Reddy, Y. ARKIVOC, 2006, (xii), 23. (b) Dunitz, J. D. Acta Cryst. 1957, 10, 307. 